\title{
INFRARED RENORMALONS AND POWER CORRECTIONS IN DEEP-INELASTIC SUM RULES*
}

\author{
Xiangdong Ji \\ Center for Theoretical Physics \\ Laboratory for Nuclear Science \\ and Department of Physics \\ Massachusetts Institute of Technology \\ Cambridge, Massachusetts 02139 \\ (MIT-CTP-2381 HEP-PH /9411312 Submitted to: Nucl. Phys. B November 1994)
}

\begin{abstract}
Infrared renormalons and $1 / Q^{2}$ power corrections in deep-inelastic sum rules are studied. The renormalization of operators with power divergence are discussed. The higher-twist terms in the operator product expansion are shown to account for the residual soft contributions survived from the Kinoshita-LeeNauenberg type of cancellation in Feynman diagrams. The presence of some degree of arbitrariness in the twist separation allows one to define the most convenient higher-twist operators suitable for a particular non-perturbative method. The discussion is focused on the Bjorken sum rule, for which the $1 / Q^{2}$ corrections are considered on a lattice.
\end{abstract}

${ }^{*}$ This work is supported in part by funds provided by the U.S. Department of Energy (D.O.E.) under cooperative agreement \#DF-FC02-94ER40818. 


\section{INTRODUCTION}

Deep-inelastic sum rules-Bjorken sum rule [1], Gross-Llewellyn Smith sum rule [2], Ellis-Jaffe sum rule [3], and a whole zoo of similar sum rules [4] - are fascinating things to study, both from theoretical and experimental points of view. These sum rules form one of the clearest tests of perturbative and non-perturbative Quantum Chromodynamics (QCD), and tests of our ability to solve the theory as well. Some of the sum rules were derived before QCD was advanted, in current algebras that the asymptotic QCD turns out to respect up to logarithms. In QCD, they can be derived using the technique of Wilson's operator product expansion (OPE) or its equivalent. The imprints of QCD in these sum rules are radiative corrections, which can be calculated perturbatively, and the higher-twist corrections that involve high-dimensional hadron matrix elements. This paper is intended to address the issue on the separation of these two effects and some related aspects in the OPE.

In order to be specific, I will center my discussion on the Bjorken sum rule, which presently is under active experimental study [5]. The sum rule is for the nucleon's spindependent structure function $g_{1}\left(x, Q^{2}\right)$ and the QCD version reads [6],

$$
\int_{0}^{1}\left(g_{1}^{p}\left(x, Q^{2}\right)-g_{1}^{n}\left(x, Q^{2}\right)\right) d x=\frac{1}{6} C_{2}\left(\alpha_{s}\left(Q^{2}\right)\right) g_{A}-\frac{4}{27} C_{4}\left(\alpha_{s}\left(Q^{2}\right)\right) \frac{\left\langle O_{4}^{\mu}\right\rangle}{Q^{2}}+\ldots,
$$

where $p$ and $n$ label proton and neutron, respectively, and $g_{A}=1.257$ is the neutron $\beta$-decay constant. $C_{i}\left(\alpha_{s}\left(Q^{2}\right)\right.$ are the coefficient functions that can be calculated in perturbation theory as power series in $\alpha_{s}\left(Q^{2}\right)$,

$$
C\left(\alpha_{s}\right)=\sum_{n=0}^{\infty} c_{n} \alpha_{s}^{n} .
$$

The second term in the right-hand-side of Eq. (1) is the twist-four correction in which $\left\langle O_{4}^{\mu}\right\rangle$ is the hadron matrix element,

$$
\left\langle P S\left|O_{4}^{\mu}\right| P S\right\rangle=2 S^{\mu}\left\langle O_{4}^{\mu}\right\rangle
$$

with

$$
O_{4}^{\mu}=\bar{u} g \tilde{F}^{\mu \alpha} \gamma_{\alpha} u-\bar{d} g \tilde{F}^{\mu \alpha} \gamma_{\alpha} d
$$

where $|P S\rangle$ is the nucleon state with momentum $P^{\mu}$ and polarization $S^{\mu}(\langle P S \mid P S\rangle=$ $\left.2 P^{0}(2 \pi)^{3} \delta^{3}(0)\right)$, and $u, d$, and $F^{\mu \nu}$ are quark and gluon fields. Both $C_{4}$ and $\left\langle O_{4}^{\mu}\right\rangle$ depend on a factorization (renormalization) scale $\mu^{2}$, however, the dependence cancels out in the product. For simplicity, I have neglected the target mass corrections and the twist-three contribution [7], both of which are present at order $1 / Q^{2}$, but inessential for the following discussion. The ellipsis denotes still higher-twist contributions that are suppressed by more powers of $1 / Q^{2}$.

It is commonly accepted that the first (twist-two) and the second (twist-four) terms as well as higher-order terms in $1 / Q^{2}$ in Eq. (1) are separately gauge invariant and finite, and hence the twist-separation is well-defined. Indeed, the leading-order coefficient function $C_{2}\left(\alpha_{s}\left(Q^{2}\right)\right.$ is often calculated in terms of the virtual Compton amplitude for a zeromomentum quark state in dimensional regularization [8], in which perturbative higher-twist 
matrix elements vanish identically, and the non-perturbative higher-twist matrix elements are known to be calculable, for instance, in lattice QCD by subtracting the corresponding perturbative matrix elements [9], or in the QCD sum rule method [10]. It turns out, however, this traditional view on the twist-separation is at best formal, and one in practice is forced to provide more details on the individual contribution. Eventually, the twist expansion has the similar degree of arbitrariness as the factorization of the coefficient functions from the operator matrix elements in individual term of the OPE [11]. This surprising feature of the twist-expansion in deep-inelastic sum rules was first recognized and studied, to the author's knowledge, by A. Mueller [12], although similar studies in vacuum current correlation functions had begun much early and the results are much more well-known 13.16 .

This paper grows out of attempts to understand the $1 / Q^{2}$ effects in the deep-inelastic sum rules [17] and Mueller's paper on ambiguity of the higher-twist contribution [12]. Since I believe most of the physicists working in deep-inelastic scattering are unaware of this issue, I intend to be pedagogical in my presentation. Therefore, the discussions in the paper are often mixed with results that are already known in the case of vacuum correlation functions. I hope the references are sufficiently clear so the reader has no confusion about what is new and what is not.

In section II, I discuss the infrared (IR) renormalons in the coefficient functions of the Bjorken sum rule. I start with an empirical observation and a calculation of a bubblechain diagram to convince the reader that the perturbation series in fact diverges. Then I state some general results known about the properties of the renormalon singularities. In section III, I consider the renormalization of operators with power-like divergence and explain why the multiplicative renormalization of log divergence cannot define the operators uniquely. The conclusion is that pure non-perturbative operators in general do not exist and higher-twist operators depend on handling of the perturbative contributions. In section IV, I derive a cut-off version of the OPE by separating the soft and hard contributions to Feynman diagrams. The result shows that the coefficient functions can be defined without IR renormalons, but depending on a momentum cut-off, and the higher-twist operators depend on the same cut-off due to perturbative power contributions. However, the sum of the twist expansion is cut-off-independent. In section V, I argue that there exists a large degree of freedom in choosing the higher-twist operators. According to this, I define a twist-four operator for the Bjorken sum rule suitable for lattice calculations. The corresponding twisttwo coefficient function is subsequently evaluated. The final section contains the discussion on the practical significance of the $1 / Q^{2}$ corrections in the Bjorken sum rule, and summary and conclusion of this paper.

\section{INFRARED RENORMALONS IN COEFFICIENT FUNCTIONS}

The IR renormalons, in the Bjorken sum rule, begin with the calculation of the coefficient function $C_{2}\left(\alpha\left(Q^{2}\right)\right)$ in dimensional regularization and minimal subtraction scheme $(\overline{\mathrm{MS}})$. According to text books [18], the coefficient functions $C_{i}$ are the coefficients of local operators in Wilson's OPE, 


$$
\int d^{4} \xi e^{i q \cdot \xi} T\left[J_{\mu}^{\mathrm{em}}(\xi) J_{\nu}^{\mathrm{em}}(0)\right]=-2 i \epsilon^{\mu \nu \alpha \beta} \frac{q_{\alpha}}{Q^{2}}\left[\frac{1}{3} C_{2}\left(\alpha_{s}\left(Q^{2}\right)\right) J_{5 \beta}(0)-\frac{8}{27 Q^{2}} C_{4}\left(\alpha_{s}\right) O_{4 \beta}+\ldots\right],
$$

where $J_{5 \beta}=\bar{u} \gamma_{\beta} \gamma_{5} u-\bar{d} \gamma_{\beta} \gamma_{5} d$ is the isovector axial current whose nucleon matrix element is $g_{A}$. Other quantities are defined following Eq. (1). Since Eq. (5) is an operator identity, it is valid when sandwiched in any state. The canonical way of calculating $C_{2}\left(\alpha_{s}\left(Q^{2}\right)\right)$ is to apply Eq. (5) to a single quark state with zero momentum. It is easy to see that the higher-twist matrix elements vanish in dimensional regularization, and $C_{2}\left(\alpha_{s}\right)$ is just the "ratio" of the quark Compton amplitude to the quark matrix element of the axial current. In fact, this is exactly what has been down in Ref. [8], where $C_{2}\left(\alpha_{s}\right)$ was calculated up to three loops,

$$
C_{2}\left(\alpha_{s}\right)=1-\frac{\alpha_{s}\left(Q^{2}\right)}{\pi}-3.58\left(\frac{\alpha_{s}\left(Q^{2}\right)}{\pi}\right)^{2}-20.21\left(\frac{\alpha_{s}\left(Q^{2}\right)}{\pi}\right)^{3}+\ldots,
$$

for three flavors. Here the renormalization scale has been chosen to be $\mu^{2}=Q^{2}$, and so there are no logarithms of type $\ln ^{k} Q^{2} / \mu^{2}$ in $c_{n}$. Notice that the coefficient $c_{n}$ is growing rapidly with $n$. An estimate for the fourth-order term gives $-130\left(\alpha_{s}\left(Q^{2}\right) / \pi\right)^{4}$ [19]. As a matter of fact, there are indications that $c_{n}$ may grow like $n$ ! for large $n$ with a fixed sign!

To see the rapid growth of $c_{n}$, consider the contribution from a $n$-loop bubble-chain diagram shown in Fig. 1a. Using dimensional regularization and modified minimal subtraction $(\overline{\mathrm{MS}}), \mathrm{I}$ find,

$$
\begin{aligned}
c_{n+1}(\text { fig.1a })= & -\frac{C_{F}}{4 \pi}\left(\frac{\beta_{0}^{\prime}}{4 \pi}\right)^{n} \operatorname{Fn}\left[2(1-\epsilon) \Gamma(2-\epsilon / 2) \sum_{k=0}^{n} C_{n}^{k}\left(-\frac{2}{\epsilon}+\gamma\right)^{k}\right. \\
& \left.\times\left(\frac{6 \Gamma^{2}(2-\epsilon / 2) \Gamma(\epsilon / 2)}{\Gamma(4-\epsilon)}\right)^{n-k} \frac{\Gamma((n+1-k) \epsilon / 2) \Gamma(1-(n+1-k) \epsilon / 2)}{\Gamma(1+(n-k) \epsilon / 2) \Gamma(3-(n+2-k) \epsilon / 2)}\right],
\end{aligned}
$$

where $C_{F}=4 / 3$ and $\beta_{0}^{\prime}=5-2 n_{f} / 3$ represents the contribution to the leading-order $\beta$ function from the one-loop gluon self-energy ( $n_{f}$ is the number of flavors). The symbol Fn refers to taking finite part of the expression, which turns out to be non-trivial. With the help of the symbolic program MAPLE, I get the following result for the rational part,

$$
\begin{array}{cc}
n & \operatorname{Fn}[\ldots] \\
20 & 2.5761918 \times 10^{19} \\
21 & 5.4100006 \times 10^{20} \\
22 & 1.1902018 \times 10^{22} .
\end{array}
$$

The irrational part that involves Reiman $\zeta$-functions and $\pi$ is small compared with the rational part, at least at small orders. Eq. (8) indicates that the contribution from the bubble-chain diagram grows like $\sim-5 \times n$ ! at large orders.

One might argue that the large coefficients from this particular diagram might cancel with those from other diagrams at the same order. However, indications are that the large $n$ behavior of $c_{n}$ for the sum of all $n$-loop diagrams is qualitatively similar to the bubble-chain diagram. To see that, consider the Borel-transformed series for $C\left(\alpha_{s}\right)$, 


$$
C(b)=\sum_{n=0}^{\infty} \frac{c_{n+1}}{n !} b^{n} .
$$

Then $C\left(\alpha_{s}\right)$ can be constructed from $C(b)$ through

$$
C\left(\alpha_{s}\right)=c_{0}+\int_{0}^{\infty} C(b) e^{-b / \alpha_{s}} d b .
$$

Then a fixed-sign $n$ ! increase is reflected by the (renormalon) poles of $C(b)$ on the positive real axis on the $b$ plane. From an analysis of the infrared physics in QCD, 't Hooft argued the existence of these poles [13]. As I shall explain later in more detail, it is the infrared behavior of QCD that leads to the appearance of the renormalon poles, and hence the divergence of the perturbation series. The locations of the poles at $b_{n}=4 \pi n / \beta_{0}(n=1,2, \ldots)$ were identified by Parisi through a study of the IR divergence of the corresponding super-renormalizable theory at $4-\epsilon$ dimension [14]. A more accurate description of the analytical structure of the IR renormalons was obtained by Mueller, who showed that the QCD radiative effects change the poles into branch points in the complex $b$ plane [16]. The leading branch point at $b_{1}=4 \pi / \beta_{0}$ gives rise to a large $n$ behavior of $c_{n}$,

$$
c_{n+1} \sim n ! n^{\gamma}\left(\frac{\beta_{0}}{4 \pi}\right)^{n},
$$

where $\gamma$ is related to $\beta_{2}$, the two-loop beta function, and $\gamma_{1}$, the one-loop anomalous dimension of the twist-four operator [12].

The existence of the IR renormalons destroys the Borel summability of the perturbation series since the integral in Eq.(10) is now ill-defined. Unlike Borel-summable series, the IR-renormalon-plagued series reflects the truly divergent nature of the expanding quantity in perturbation theory; the divergence has nothing to do with the choice of the expansion parameter. Since at large $Q^{2}$ higher-twist terms are exponentially small compared with the terms in the leading-twist, and since different ways of regularizing the Borel integral in Eq. (10) produce an uncertainty of order of $1 / Q^{2}$, the the nature of the OPE clearly deserves a more careful examination [15].

\section{COMPOSITE OPERATORS WITH POWER DIVERGENCE}

Composite operators in QCD have a special importance: The chiral symmetry breaking is characterized by a non-vanishing expectation value of $\bar{\psi} \psi$ in the vacuum. The electroweak couplings of quarks are through vector and axial-vector currents $\bar{\psi} \gamma^{\mu} \psi$ and $\bar{\psi} \gamma^{\mu} \gamma_{5} \psi$. The trace anomaly of the energy-momentum tensor are proportional to $F^{2}=F^{\alpha \beta} F_{\alpha \beta}$, and the axial anomaly is proportional to $F^{\alpha \beta} \tilde{F}_{\alpha \beta}$. Finally, Wilson's operator product expansion employs myriad composite operators.

Composite operators are badly divergent in field theory, and are not made finite through the standard renormalization of Green's functions [18]. Rather, they are renormalized individually and have their own renormalization scale dependence. For these reasons, composite operators are normally not physical observables. When they do appear as a part of a physical quantity, the renormalization scale is determined either by physics consideration or cancelled by the scale-dependence of other quantities. 
According to the divergence behaviors of composite operators in QCD, they can be classified into three categories (I disregard operators with vanishing physical matrix elements, which include BRST-exact operators and the equations of motion operators [20]):

- Finite operators: These include vector and non-singlet axial-vector currents of quarks and their divergence like $\bar{\psi} m \psi$, the energy-momentum tensor, the angular momentum density, the topological charge density $F \tilde{F}$, etc. These operators are associated with the symmetries of the QCD lagrangian, and are physical observables of the system.

- Operators with logarithmic divergence: All twist-two and twist-three operators have logarithmic, and only logarithmic, divergence. Here I restrict myself to just local operators. Let me remind the reader that the twist of an operator is defined by the difference of the dimension of the operator and its rank of spin in representations of the Lorentz group. Although the notion of twist arises from study of deep-inelastic scattering, no reference here is needed to this application. [All operators can be classified in terms of twist because of the Lorentz symmetry.] The twist-two and twist-three operators have just logarithmic divergence because their matrix elements in physical states are dimensionless quantities. They cannot mix under renormalization with any operators of lower dimensions. However, they can mix among themselves, yielding a dimensionless mixing matrix. The renormalized operators depend on the genuine renormalization scale $\mu^{2}$.

- Operators with power divergence: Operators of twist-four and higher normally have power divergence. Exceptions include finite operators discussed above and operators with special symmetry properties. The simplest example of operators with power divergence is $F^{2}$. One might argue that since the energy-momentum tensor is a finite operator and $F^{2}$ appears in its trace (trace anomaly), so $(\beta(g) / 2 g) F^{2}$ must be a finite operator. This is true as long as one is talking about the difference of the matrix elements in the excited states of QCD and the vacuum. Since the vacuum energymomentum density is not a physical observable, $(\beta(g) / 2 g) F^{2}$ in the vacuum needs not to be finite (the proper normal ordering of the operator is always implied here.)

Once an operator has quadratic or quartic or higher-order divergence, they can mix under renormalization with lower-twist operators. For instance, $F^{2}$ can mix with the trivial operator 1 , and $\bar{\psi} \tilde{F}^{\mu \nu} \gamma_{\nu} \gamma_{5} \psi$ can mix with $\bar{\psi} \gamma^{\mu} \psi$, etc. As shall become clear soon, these mixings have important implications about the twist separation in the OPE.

Let me consider the renormalization of power-divergent operators in dimensional regularization. Although one cannot do non-perturbative calculations in this regularization, the coefficient functions in the leading-twist are normally calculated in the scheme [21]. Since perturbative QCD does not have any mass scale, the mixing of higher-twist operators with lower-twist ones vanishes identically. [Integrals of type $\int d^{d} k / k^{m}$ are taken to be zero.] Only logarithmic divergence appears in the matrix elements of the operators, which can be renormalized in a standard way. Thus it seems that composite operators can be defined up to their logarithmic divergence and are devoid of any power-dependent perturbative contributions.

This standard treatment of composite operators in dimensional regularization is in fact deceptive. In principle, infrared and ultraviolet physics are entirely different and shall not be 
mixed. However, the statement $\int d^{d} k / k^{2}=0$, for instance, has a ridiculous implication that the ultraviolet divergence is cancelled by the integration in the infrared region! Not only the integration is not reliable in the low $k^{2}$ region due to non-perturbative physics, but also is finite. Thus the implied cancellation can never occur in reality. Actually, the ultraviolet power divergence should not be thrown away like this because they dictate the correct operator mixing. Furthermore, a perturbative calculation in the low $k^{2}$ region generates IR renormalons just like the coefficient functions discussed in the last section. Thus $\int d^{d} k / k^{2}=$ 0 is mathematically convenient, but physically unwanted.

Indeed, in a study of the two-dimensional $\mathrm{O}(\mathrm{N})$ non-linear sigma model, David found that the composite operators with power divergence are not well-defined in dimensional regularization [15]. He calculated the spin-wave condensate in the physical vacuum using the regularization (which is possible in $1 / N$ expansion) and found that the result is not unique. His argument for the presence of the ambiguity in the composite operator is subtle and roughly goes like this: When $\epsilon=4-d$ is kept finit in dimensional regularization, the higher-twist matrix element has a series of poles on the positive real-axis of the $\epsilon$ plane starting from $\epsilon=$ the degree of divergence of the operator. The poles are present because at these $\epsilon$, one cannot distinguish a higher-twist contribution from a lower-twist one. These poles make the limit of $\epsilon \rightarrow 0$ ambiguous, depending on whether one takes $\operatorname{Im} \epsilon>$ or $<0$.

The interpretation of David's ambiguity is that composite operators in dimensional regularization have IR renormalons. They were not there originally, but appear when the scheme insists to treat the contribution in the soft momentum region in perturbation theory. Those perturbative soft contributions are needed to cancel the ultraviolet power divergence because the relations like $\int d^{d} k / k^{m}=0$ are used. Consequently the power divergence is killed with a price: emergence of IR renormalon singularities. It is these singularities that cause the ambiguity in David's calculation. [An explicit example of the renormalons in matrix elements is provided in the next section in Eq. (20)] Thus in dimensional regularization, in addition to subtract the logarithmic divergence of an operator, one needs to specify how the IR renormalons are regulated.

What if one uses other regularization schemes, such as the scheme with a momentum cut-off? In a cut-off scheme, composite operators are finite, as long as the cut-off is kept finite. The operators are also free of any IR renormalon singularities. Their physical matrix elements depend on two scales: the non-perturbative scale generated from the breaking of the scale symmetry in the non-perturbative QCD [22] and the perturbative scale that is served as a cut-off for both the log and power divergences. If the degree of divergence of an operator is $d_{O}$, its matrix element goes like the $d_{O}$ power of the cut-off when the cut-off approaches infinity. In light of dimensional regularization, it is tempting to define a pure non-perturbative matrix element by subtracting off the corresponding perturbative matrix element. Although the cut-off dependence now disappears, the IR renormalon singularities are introduced through perturbative matrix elements. Thus a pure non-perturbative matrix element is again not well-defined.

To summarize the above discussion, operators with power divergence cannot be specified uniquely after subtracting their logarithmic divergence in dimensional regularization. One must specify in addition how the IR renormalons are regularized, or, equivalently, how the limit $\epsilon \rightarrow 0$ is taken. In a cut-off regularization, the operators are cut-off dependent, independent of how the logarithmic divergence is regulated. For example, $\beta(g) / 2 g F^{2}$ has 
no logarithmic divergence at all but still is cut-off dependent. One important consequence of this is that there are no physically well-defined higher-twist contributions in the OPE.

Since the physical quantity that is expanded in the OPE is well-defined, the ambiguity in defining higher-twist operators must be correlated with the infrared renormalon singularities in the coefficient functions. In the 2D non-linear sigma model, David showed that the different choices for regularizing the Borel integral and defining the composite operators give the same final result. Thus despite the existence of arbitrariness in defining the contributions of different twists, the spirit of OPE is unspoiled.

In fact, the situation with the twist separation is analogous to the factorization of collinear singularities in the OPE [18]. There the leading-twist contribution in the OPE can be written as a product of the coefficient functions and the hadron matrix elements. The coefficient functions depend on the factorization scale because they are obtained by subtracting off collinear divergence in a single parton scattering. The matrix elements are scale-dependent because the local operators are renormalized operators disposed of ultraviolet divergence. The factorization scale controls how much physics is considered to be perturbative and how much is non-perturbative. Their product is scale-independent.

\section{OPERATOR PRODUCT EXPANSION IN A CUT-OFF SCHEME}

According to the previous two sections, the traditional recipe for the OPE is at best formal: The coefficient functions are intrinsically divergent and higher-twist operators are illdefined after removing the logarithmic divergence. However, both problems have a common origin - the IR renormalons. The existence of the renormalons makes the notion of twist separation procedure-dependent, however, specifics cancel in the physical sum. Specifically, one needs to define the limit $\epsilon \rightarrow 0$ in higher-twist operators consistent with handling of the Borel integral for the coefficient functions [15]. However, the approach is hardly useful in practical applications. First, no one knows how to calculate the non-perturbative matrix elements of higher-twist operators in dimensional regularization in QCD. Second, despite tremendous progress made recently in calculating the QCD perturbative series to higher orders [19], it remains a formidable task to calculate the exact behavior of a infrared renormalon series. Lastly, even all the above are possible, the regularization does not seem to have clear physical motivation. [The author noticed a number of other proposals in the literature for regularization the infrared renormalons [12,23.]

To my opinion, a better approach to the OPE, which was advocated by the former ITEP group [24,25], and was first taken seriously by Mueller [16], is to consider the twist expansion entirely from the point of view of Feynman diagrams. Imagine a set of Feynman diagrams completely define a physical cross section or a correlation function in QCD. Often in accompany with a high-energy subdiagram, there are soft subprocesses mediated by lowenergy quarks and gluons, which result in soft (infrared) and collinear (mass) divergence in perturbative calculations [18]. In many physical observables, the soft divergence cancel according to equivalent of the Kinoshita-Lee-Nauenburg theorem [26], and the collinear divergence can be factorized into hadron matrix elements, which in the end are replaced with proper non-perturbative ones [11]. The remaining part is calculable as a perturbation series in the strong coupling constant $\alpha_{s}$ and is called infrared safe. The classical examples of the perturbative-calculable quantities include the total rate for $e^{+} e^{-} \rightarrow$ hadron, the coefficients 
in Wilson's operator product expansion, and radiative corrections to jet production, DrellYan cross section, etc.

However, it was pointed out by Shifman, Vainshtein, and Zakharov (SVZ) that using "infrared-safe" as a criteria to decide a perturbative calculation is inadequate [24. They argued, in the case of current correlation functions in the vacuum, that the part of Feynman diagrams involving low-virtuality quarks and gluons cannot be calculated perturbatively despite such a calculation contains no infrared divergence and the contribution might be small. A legitimate calculation for the part must be done non-perturbatively, by using, in their case, various vacuum condensates. These vacuum condensates correspond to the higher-twist terms in Wilson's OPE. Thus, the role of higher-twist contributions is to take into account properly the residual soft contributions in Feynman diagrams after the KLN type of cancellation.

Thus to devise a twist expansion, one can start by separating the hard and soft contributions in Feynman diagrams. This is precisely what Mueller did for vacuum correlation functions [16]. In this way, he obtained coefficient functions that are entirely perturbative and free of IR renormalons, and the higher-twist operators that are free of ultraviolet divergence due to an explicit cut-off. Both contributions now depends on the twist-separation scale but the sum does not. Notice the separation scale here plays the same role as the prescriptions of regularizing the higher-twist operators in David's paper. In this section, I follow Mueller's approach in Ref. [16] and attempt to construct a twist expansion for the Bjorken sum rule. To motivate the approach, let me come back to the physical origin of the renormalon singularities in the perturbation series.

The appearance of $n$ ! in the coefficient functions is due to loop integrations in small momentum regions in Feynman diagrams [16,27. In fact, consider a one-loop Feynman diagram with the gluon momentum $k$ (chosen to be the loop momentum). Replace the gluon coupling $\alpha_{s}\left(Q^{2}\right)$ (again, $\mu^{2}=Q^{2}$ ) with a running coupling $\alpha_{s}\left(k^{2}\right)$,

$$
\alpha_{s}\left(k^{2}\right)=\frac{\alpha_{s}\left(Q^{2}\right)}{1+\alpha_{s}\left(Q^{2}\right) \frac{\beta_{0}}{4 \pi} \cdot \ln \frac{k^{2}}{Q^{2}}}
$$

Then the small $k^{2}$ integration is questionable because where the perturbative expression for $\alpha_{s}\left(k^{2}\right)$ breaks down. [Notice the existence of the familar Landau ghost pole at $k^{2}=$ $Q^{2} \exp \left(-\alpha_{s}\left(Q^{2}\right) \beta_{0} /(4 \pi)\right)$.] On the other hand, when expanding $\alpha_{s}\left(k^{2}\right)$ in terms of $\alpha_{s}\left(Q^{2}\right)$,

$$
\alpha_{s}\left(k^{2}\right)=\alpha_{s}\left(Q^{2}\right) \sum_{n}(-1)^{n}\left(\frac{\alpha_{s}\left(Q^{2}\right) \beta_{0}}{4 \pi}\right)^{n} \ln ^{n}\left(\frac{Q^{2}}{k^{2}}\right),
$$

the $n$-th term represents a legitimate partial contribution from the $n$-th order Feynman diagrams. When it is inserted in the loop integral, the repeated partial integrations give $n$ !. A simple examination reveals that most of the contribution comes from the region of the integration $k^{2} \sim Q^{2} e^{-n}$, where the logarithms become large. Therefore the divergence of the perturbation theory is caused by illicit perturbative calculations in the soft regions where perturbative quarks and gluons are ill-defined. Although order by order, perturbative contributions from these regions are finite, they diverge as $n \rightarrow \infty$ irrespetive the value of $Q^{2}$. Thus, a physically motivated OPE shall incorporate the perturbative soft (infrared) contributions in the higher-twist terms. 
To identify such contributions in the Bjorken sum rule, consider the one-loop contribution to the coefficient function shown in Fig. 2. The one-loop integrations are free of ultraviolet divergence after the standard QCD renormalization. The external quark momentum is set to zero after the collinear singularity in the box diagram (Fig. 2d) is subtracted by the one-loop matrix element of the axial current [21]. Now focus on contributions from the region where the gluon momentum is soft ( $k^{2}$ is small). In this region, one can expand the integrand in $k^{2} / Q^{2}$ and keep the leading-order contribution. Applying an upper cut-off $\Lambda^{2}$ on the gluon virtuality, I find the perturbative part of $c_{1}$ from the soft region,

$$
\begin{aligned}
\Delta c_{1}^{\text {soft pert. }} & =\frac{8 g^{2}}{3 Q^{2}} C_{F} \int_{0}^{\Lambda^{2}} \frac{d^{4} k}{(2 \pi)^{4}} \frac{-i}{k^{2}} \\
& =-\frac{8}{9} \frac{\alpha_{s}\left(Q^{2}\right)}{\pi} \frac{\Lambda^{2}}{Q^{2}},
\end{aligned}
$$

where in the second line have rotated the integration to the Euclidean space. The factor $8 / 3$ in the first line is the sum of 2,1 , and $-1 / 3$, coming from diagrams a), b), and c) in Fig. 2, respectively. The cut-off $\Lambda^{2}$ should be on the order of 0.5 to $1 \mathrm{GeV}^{2}$, above which perturbative calculation is justifiable. Clearly, the contribution in Eq. (14) must subtracted from $c_{1}$ and the corresponding non-perturbative contribution should be added as a higher-twist contribution.

To find the correct non-perturbative contribution, I re-examine the one loop diagrams in Fig. 2. When the gluons are soft, we cannot contract the field operators in the perturbative vacuum, instead we must keep them and evaluate the matrix elements in the non-perturbative zero-momentum quark state. [The same thing can be said of any quark lines that carry soft momenta.] For example, the diagrams (b) and (c) in Fig. 2 contribute to the quark Compton amplitude,

$$
T_{q}^{\mu \nu}=\int \frac{d^{4} k}{(2 \pi)^{4}} \frac{d^{4} k_{1}}{(2 \pi)^{4}} H^{\mu \nu \alpha}\left(k, k_{1}\right) S_{\alpha}\left(k, k_{1}\right),
$$

where $k$ and $k_{1}$ are quark and gluon momenta, respectively, with Euclidean $k_{1}^{2}$ restricted to $\leq \Lambda^{2} . H^{\mu \nu \alpha}$ represents part of the diagrams above the dashed lines and is perturbative. $S_{\alpha}$ represents that below and can be obtained from the first-order calculation of

$$
S_{\alpha}\left(k, k_{1}\right)=\int d^{4} \xi d^{4} \xi_{1} e^{i k \cdot \xi} e^{i k_{1} \cdot \xi_{1}}\left\langle q(0)\left|\mathrm{T} \bar{\psi}(\xi) A_{\alpha}\left(\xi_{1}\right) \psi(0)\right| q(0)\right\rangle
$$

where $\psi$ and $A^{\mu}$ are quark and gluon field operators and $|q(0)\rangle$ is a zero-momentum quark state. Thus the one-loop diagrams arise from a perturbative expansion of the zeromomentum-quark wave function, which of course is incorrect as the gluon momentum becomes soft. However, so long as one refrains from such a perturbative expansion, one gets the correct contribution from the soft-gluon region.

To proceed, I use the technique of the collinear expansion as explained in full details in Ref. 28]. The result is,

$$
T_{q}^{\mu \nu}=2 i \epsilon^{\mu \nu \alpha \beta} \frac{8 q_{\alpha}}{9 Q^{4}}\left\langle q(0)\left|O_{4 \beta}^{\text {cut }}\right| q(0)\right\rangle
$$

where, 


$$
\begin{aligned}
O_{4 \beta}^{\text {cut }}= & \int_{k_{1}^{2}, k_{2}^{2}<\Lambda^{2}} \frac{d^{4} k_{1}}{(2 \pi)^{4}} \frac{d^{4} k_{2}}{(2 \pi)^{4}} d^{4} \xi_{1} d^{4} \xi_{2} e^{i k_{1} \cdot \xi_{1}} e^{i k_{2} \cdot \xi_{2}} \\
& \times \bar{\psi}(0)\left[\gamma_{\beta} \gamma_{5} i D_{\perp}\left(\xi_{1}\right) \cdot i D_{\perp}\left(\xi_{2}\right)+i \epsilon^{\alpha \eta \gamma \delta} p_{\gamma} n_{\delta} \gamma_{\beta} i D_{\alpha}\left(\xi_{1}\right) i D_{\eta}\left(\xi_{2}\right)\right] \psi(0)
\end{aligned}
$$

where $p$ and $n$ are light-like vectors that are introduced as a part of the coordinate basis. Clearly, as $\Lambda^{2} \rightarrow \infty, O_{4 \beta}^{\text {cut }} \rightarrow O_{4 \beta}$, the local twist-four operator appearing in Eq. (5). For a fixed $\Lambda^{2}, O_{4 \beta}^{\text {cut }}$ is a non-local operator. It is simple to show that at the one-loop order the perturbative calculation of the twist-four matrix element reproduces the soft perturbative contribution to the coefficient function in Eq. (14).

The above result tells us several things about the operator product expansion in Eq. (5). First, the higher-twist operator has an upper cut-off in the Fourier components for the gluon fields, and thus is finite. To fully include the non-perturbative effect, the cut-off $\Lambda^{2}$ must be larger than any non-perturbative scale, such as $\Lambda_{\mathrm{QCD}}$. Second, when quarks and gluons have virtuality greater than $\Lambda^{2}$, their effects are perturbative and are included in the coefficient functions. The separation of the two effects are scale-dependent, but the sum is not. Lastly, although I discussed only one-loop diagrams with one soft-gluon, the nonperturbative twist-four contribution takes care of all Feynman diagrams with one soft-gluon. Diagrams with one soft-quark line do not contribute at the twist-four level [29]. Diagrams with two soft-gluon or quark lines are related to the twist-six terms, which are beyond the scope of this paper.

Clearly, when taking $O_{4 \beta}^{\text {cut }}$ as a definition of higher-twist operator, the corresponding coefficient function in Eq. (5) shall be modified,

$$
C_{2}\left(\alpha_{s}\right)=C_{2 \text { pert }}\left(\alpha_{s}\right)+\frac{8}{9} C_{4}\left(\alpha_{s}\right) \frac{\left\langle O_{4 \beta}^{\text {cut }}\right\rangle_{\text {pert }}}{Q^{2}}
$$

where $C_{2 \text { pert }}\left(\alpha_{s}\right)$ the usual perturbation series in Eq. (2),$C_{4}\left(\alpha_{s}\right)$ is the coefficient function for the twist-four operator. The perturbative matrix element of $O_{4 \mu}^{\text {cut }}$ is to be evaluated in a zeromomentum quark state and is expected to have IR renormalons. Since soft contributions are subtracted in Eq. (19), we expect that $C_{2}\left(\alpha_{s}\right)$ is free of the leading IR renormalon singularity. However, the renormalon poles at $4 \pi n / \beta_{0}$ with $n \geq 2$ are still present. To subtract them, one must consider the two-loop contributions to $C_{2 \text { pert }}\left(\alpha_{s}\right)$ and the twist-six operators.

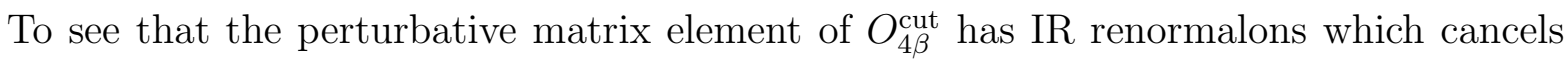
the leading IR renormalon in $C_{2 \text { pert }}\left(\alpha_{s}\right)$, I consider again the bubble-chain diagram shown in Fig. 1a. The corresponding contribution to the matrix element is shown in Fig. 1b. A simple calculation shows,

$$
\begin{aligned}
\left\langle O_{4 \beta}^{\text {cut }}\right\rangle_{\text {pert }}(\text { Fig.1b })= & \frac{\alpha_{s}\left(Q^{2}\right)}{4 \pi} C_{F}\left(\frac{\beta_{0}^{\prime} \alpha_{s}\left(Q^{2}\right)}{4 \pi}\right)^{n} \operatorname{Fn}\left[3 \Lambda^{2} \sum_{k=0}^{n} C_{n}^{k} \frac{1}{1-\epsilon(n-k) / 2}\right. \\
& {\left.\left[\frac{6 \Gamma(2-\epsilon / 2)^{2} \Gamma(\epsilon / 2)}{\Gamma(4-\epsilon)}\right]^{n-k}\left(-\frac{2}{\epsilon}+\gamma\right)^{k}\left(\frac{Q^{2}}{\Lambda^{2}}\right)^{\epsilon(n-k) / 2}\right] }
\end{aligned}
$$

Thus the subtraction is both $\Lambda^{2}$ and $Q^{2}$ dependent. If I fix $\Lambda^{2}$ at $1 \mathrm{GeV}^{2}$, the subtraction is maximum at $Q^{2}=1 \mathrm{GeV}^{2}$. As $Q^{2}$ increase, the subtraction becomes smaller, indicating 
less contributions in $c_{n}$ from the non-perturbative region. For large $n$, the subtraction is a exceedingly slow-varying function of $Q^{2}$. In fact, defining $y=\Lambda^{2} / Q^{2}$, I find at $n=20$,

$$
\begin{array}{cc}
y & 2 \mathrm{Fn}[\ldots] /\left(3 Q^{2}\right) \\
0.1 & 2.5761951 \times 10^{19} \\
0.01 & 2.5761877 \times 10^{19} \\
0.001 & 2.5755872 \times 10^{19}
\end{array}
$$

Comparing with the result from Eq. (8), the subtraction is accurate up to one part per million for $y=0.1$. As $n \rightarrow \infty$, the large perturbative contributions to $C_{2 \text { pert }}$ are entirely subtracted, independent of $Q^{2}$ and $\Lambda^{2}$.

\section{TWIST-FOUR CONTRIBUTION IN POINT-SPLITTING REGULARIZATION}

The cut-off scheme discussed in the previous section best illustrates the goal of the OPE: separating the perturbative and soft (infrared) contributions in a process. In analogy with the factorization of the collinear singularities at each twist, the twist separation is not unique. In the cut-off scheme, this is shown by dependence of higher-twist operators on the cut-off scale $\Lambda$. The size of $\Lambda$ must be large enough so that all non-perturbative physics is covered by higher-twist matrix elements, and at the same time shall be small enough that the pure perturbative physics is included in the coefficient functions of the leading-twist terms. In QCD, one believes that such scale exists around $1 \mathrm{GeV}^{2}$.

In view of what twist-expansion accomplishes, I argue that there exists a large degree of arbitrariness in choosing a cut-off version of the twist-four operator $O_{4 \mu}$ : The specific cut-off scheme that I discussed before is only one example. As long as it fully incorporates the non-perturbative physics, as long as it approaches $O_{4 \mu}$ when cut-off is let go to infinity, any finite, non-local operator with right dimension and quantum numbers is a good twistfour operator. Once a choice is made, the coefficient function of the twist-two term can be calculated through applying the OPE to, say, a perturbative zero-momentum quark state. Thus the computation of the coefficient functions goes hand in hand with the choices of the higher-twist operators.

The freedom of twist-separation is very useful in practical applications. The twist-four operator in the cut-off scheme is quite complicated, not suitable for non-perturbative methods. In what follows, I seek a definition of the twist-four operator convenient for lattice calculations, because the lattice is the only practical means at present for a non-perturbative calculation. Following the definition, I calculate the corresponding twist-two coefficient function. The approach here can be generalized straightforwardly to the application of the OPE to the QCD sum rules, which will be discussed in a separation publication [30].

I choose to define composite operators in Euclidean space, in which the lattice realization is usually made. Imagine the space-time is discretized into a lattice with hypercubes; the lattice spacing is $\pi / \Lambda$. The quark fields are assigned at every lattice point and gluon fields are represented by gauge links with

$$
U_{\mu}(n)=\mathcal{P} e^{-i g(\pi / \Lambda) \int_{0}^{1} A_{\mu}(n+x \hat{\mu}) d x}
$$


representing a link, starting at the discrete point $n$ and ending at a neighbouring lattice point in the direction $\hat{\mu}$. $\mathcal{P}$ is the path-ordering operator. I define an operator on lattice,

$$
O_{4 \alpha}\left(\Lambda^{2}, n\right)=i \frac{\Lambda^{2}}{4 \pi^{2}} \epsilon_{\alpha \beta \mu \nu} \bar{\psi}(n)\left[U_{\mu}(n) U_{\nu}(n+\hat{\mu})-U_{\nu}(n) U_{\mu}(n+\hat{\nu})\right] \gamma_{\beta} \psi(n+\hat{\mu}+\hat{\nu})+\text { h.c. }
$$

where h.c. represents the hermitian conjugation term. The definitions of various Euclidean variables follow those of Rothe [31]. It is simple to show that in the limit of $\Lambda^{2} \rightarrow \infty$, the operator goes to $O_{4 \alpha}$. Under the standard lattice gauge transformation,

$$
\begin{aligned}
\psi(n) & \rightarrow u(n) \psi(n) \\
U_{\mu}(n) & \rightarrow u(n) U_{\mu}(n) u^{\dagger}(n+\hat{\mu})
\end{aligned}
$$

where $u(n)$ is an element of the color $\mathrm{SU}(3)$ group, the operator is gauge invariant. Since the gluon fields are integrated along a straight path in a distance of $\pi / \Lambda$, the large momentum components of the fields have effectively been cut off - the operator is finite.

A few clarification is in order. First of all, I have said nothing about lattice QCD so far. I use the lattice as a tool to motivate a non-local, gauge invariant twist-four operator. Thus the actual quark and gluon fields and the location $n^{\mu}$ of the plaquets can still be regarded as continuous. [The method of defining finite operators is very similar to the well-known Schwinger's method of splitting the fermion fields in a current.] Second, the operator is non-local in Euclidean time, thus one has to rotate all loop integrals to Euclidean space in a perturbative calculation. Lastly, the operator has no complete Lorentz symmetry. However, the violation occurs at the scale of $\Lambda$ around which physics is perturbative. When its matrix elements are used in conjunction with the coefficient function defined below, the symmetry breaking effects largely cancel.

With the above definition of the twist-four operator, one can calculate the nonperturbative matrix element in a polarized nucleon state in lattice QCD. For instance, by choosing a series of lattice spacings $a_{n}=\pi /(n \Lambda)(n=1,2, \ldots)$, one can construct the operator from lattice variables almost trivially. By comparing a series of calculations with different $n$, one can extract the matrix element in the continuum limit. [Note that the scale $\Lambda$ in the operator shall not be changed as the lattice spacing $a$ approaches zero. The presence of this scale ensures the existence of the finite matrix element in the limit.] The lattice calculation is currently been persued by the MIT lattice group [32].

What remains is to calculate the corresponding coefficient function of the twist-two operator. As usual, I sandwich the OPE in a zero-momentum quark state and calculate everything using dimensional regularization, including the perturbative matrix element of the twist-four operator. At one-loop level, the matrix element is,

$$
\begin{aligned}
\left\langle q(0)\left|O_{4}^{\mu}\left(\Lambda^{2}\right)\right| q(0)\right\rangle & =g^{2} C_{F} \frac{\Lambda^{2}}{\pi^{2}} \sum_{\mu \nu} \int \sin \left(\frac{k_{\mu} \pi}{\Lambda}\right) \sin \left(\frac{k_{\nu} \pi}{\Lambda}\right) \frac{k_{\mu}}{k_{\nu}} \frac{1}{k^{4}} \frac{d^{4} k}{(2 \pi)^{4}} \bar{u} \gamma^{\mu} \gamma^{5} u \\
& =\frac{2 \alpha_{s} \Lambda^{2}}{\pi^{2}} \bar{u} \gamma^{\mu} \gamma^{5} u .
\end{aligned}
$$

The calculation is done with Feynman rules in Euclidean space. From this we get the one-loop contribution to the coefficient function, 


$$
c_{1}=-\frac{1}{\pi}\left(1-\frac{16 \Lambda^{2}}{9 \pi Q^{2}}\right)
$$

The power term here is quite close to the power term in the cut-off scheme in the previous section. I emphasize again that this coefficient function must be used together with the specific twist-four operator in Eq. (23).

Another way to define the twist-four operator is to let the non-locality of the operator in Eq.(23) $\Lambda$ be the same as the lattice spacing $a$, approaching zero in the continuum limit. Then the lattice matrix element diverges like $1 / a^{2}$. A finite twist-four operator can be defined by subtracting from the original operator its perturbative matrix element on the lattice, with a low momentum cut-off in loop integrals. Thus the twist-four contribution is again free of the IR renormalons but still depends on the infrared cut-off. Unfortunately, it is difficult to do perturbative calculations with a nucleon state. However, the idea is clearly applicable to vacuum condensates in the QCD sum rules [9,30].

\section{COMMENTS AND CONCLUSIONS}

According to the discussions in the previous sections, the leading-twist contribution to the Bjorken sum rule can still be regarded as a power series in $\alpha_{s}\left(Q^{2}\right)$. However, the coefficient in each order is now not just a numerical number; it contains the subtraction of the soft contributions that are represented by a power series in $\Lambda^{2} / Q^{2}$. The most important soft-subtractions are those nominally suppressed by one power of $1 / Q^{2}$. As the order of the perturbation $n \rightarrow \infty$, the subtraction grows like $n$ !, independent of the value of $Q^{2}$ and the subtraction scale $\Lambda^{2}$. Therefore, the series in $\alpha_{s}$ would converge if other renormalons singularities and sources of divergent factors did not exit. [It is known that ultraviolet renormalons and the singularities in the Borel plane caused by instanton-anti-instanton pairs still make the series asymtotic, but I ignore them here.]

Thus to the accuracy of $1 / Q^{2}$, one can successively take into account the QCD corrections in the following way. First calculate the coefficient function $C_{2}\left(\alpha_{s}, \Lambda^{2} / Q^{2}\right)$ in powers of $\alpha_{s}$. At each other, the $1 / Q^{2}$ subtraction must be calculated explicitly. At lower orders, the subtraction is smaller than the pure loop contributions. However, the former must be kept to maintain the accuracy. As $n$ increases, both the loop contributions and subtractions decrease initially, and their difference also decreases. If the difference becomes the size of the non-perturbative $1 / Q^{2}$ terms, the latter must be included. With the further increase of $n$, both the perturbative contributions and their subtractions start to grow. However, the cancellation now becomes more complete and the residual remains small. The first few terms of the Bjorken sum rule is,

$$
\begin{aligned}
\int_{0}^{1}\left(g_{1}^{p}\left(x, Q^{2}\right)-g_{1}^{n}\left(x, Q^{2}\right)\right) d x= & \frac{g_{A}}{6}\left(1-\frac{\alpha_{s}\left(Q^{2}\right)}{\pi}\left(1-\frac{16 \Lambda^{2}}{9 \pi Q^{2}}\right)-3.58\left(\frac{\alpha_{s}}{\pi}\right)^{2}\left(1-\eta \frac{\Lambda^{2}}{Q^{2}}\right)-\ldots\right) \\
& -\frac{4}{27} \frac{\left\langle O_{4}^{\mu}\left(\Lambda^{2}\right)\right\rangle}{Q^{2}}+\ldots
\end{aligned}
$$

The soft contribution in the two-loop diagrams, denoted by $\eta$, needs to be calculated.

The non-perturbative twist-four contributions can be calculated on lattice, as explained in the last section. In the previous studies, the twist-four matrix elements have been evaluated in the MIT bag model and the QCD sum rule method. The bag model calculation has 
no explicit cut-off dependence [7], which is a common problem for model estimates. Empirically, one may regard the cut-off being somewhere from 0.5 to $0.8 \mathrm{GeV}^{2}$ [33]. The QCD sum rule calculations for the higher-twist matrix elements are clearly questionable [10]. As I explained in sec. III, the operators used in these calculations are not well-defined.

The IR renormalons exist in all perturbation series in QCD. The issue is closed related to the non-perturbative power corrections. Unlike the case of QED, the power corrections are an important subject in perturbative QCD. For instance, a precision determination of the strong coupling constant clearly needs a correct understanding of the higher-twist effects. In fact, already at present, the effects become the dominating theoretical uncertainty in some of the data analysis [34]. The framework described in this paper provides one way get the problem under control.

To summarize, we have studied in this paper the IR renormalons and $1 / Q^{2}$ corrections in deep-inelastic sum rules. The result shows that the non-perturbative $1 / Q^{2}$ corrections depend on specific choices of the regularized higher-twist operators. I have proposed to define a twist-four operator for the Bjorken sum rule, which is suitable for lattice calculations. The coefficient functions for the leading-terms can only be calculated after the higher-twist operators are defined. Although most of the discussions in the paper are made for the Bjorken sum rule, all the results are clearly applicable to all deep-inelastic sum rules.

I wish to thank A. Mueller for many useful discussions, comments, and suggestions. Discussions with K. Johnson, W. Lin, J. Negele, B. Schreiber, U. Wiese are also acknowledged. 


\section{REFERENCES}

[1] J. Bjorken, Phys. Rev. 148 (1966) 1467.

[2] D. J. Gross and C. H. Llewellyn Smith, Nucl. Phys. B14 (1969) 337.

[3] J. Ellis and R. L. Jaffe, Phys. Rev. D9 (1974) 1444.

[4] See for example, F. J. Yndurain, Quantum Chromodynamics, Spring-Verlag, 1983.

[5] The SMC Collaboration, B. Adeva et al., Phys. Lett. B302 (1993) 533; ibid, B329 (1994) 399; The E142 Collaboration, P. L. Anthony et al., Phys. Rev. Lett. 71 (1993) 959;

[6] E. V. Shuryak and A. I. Vainshtein, Nucl. Phys. B199 (1982) 451; R. L. Jaffe and M. Sodate, Phys. Rev. D26 (1982) 49.

[7] X. Ji and P. Unrau, Phys. Letts. B333 (1994) 228.

[8] S. Larin and J. Vermaseren, Phys. Lett. B259 (1991) 345.

[9] T. Banks et al., Nucl. Phys. B190 (1981) 692; A. DiGiacomo and Rossi, Phys. Lett. 100B (1981) 481, 108B(1982) 327.

[10] I. I. Balitsky, V. M. Braun, and A. V. Kolesnichenko, Phys. Lett. B242 (1990) 245; erratum: ibid, B318 (1993) 648; G. G. Ross and R. G. Roberts, Phys. Lett. B322 (1994) 425.

[11] See, for example, J. Collins, G. Sterman, D. Soper, in Perturbative Quantum Chromodynamics, ed. A. Mueller, World Scientific, Singapore, 1989.

[12] A. H. Mueller, Phys. Letts. B308 (1993) 355.

[13] G. 't Hooft, in: The whys of subatomic physics (Erice, 1977), ed. A. Zichichi (Plenum, New York, 1977).

[14] G. Parisi, Phys. Lett. B76 (1978); Nucl. Phys. B150 (1979) 163.

[15] F. David, Nucl. Phys. B234 (1984) 237; B263 (1986) 637.

[16] A. H. Mueller, Nucl. Phys. B250 (1985) 327.

[17] X. Ji and P. Unrau, MIT-CTP preprint No. 2348, 1994.

[18] T. Muta, Fundations of Quantum Chromodynamcs, World Scientific, Singapore, 1987.

[19] A. L. Kataev, and V. Starshenko, CERN-TH-7198-94, hep-ph/9405249.

[20] J. Collins, Renormalizations, Cambridge Univ. Press, Cambridge, 1984.

[21] W. A. Bardeen, A. J. Buras, D. W. Duke, and T. Muta, Phys. Rev. D18 (1978) 3998.

[22] R. T. Crewther, Phys. Rev. Letts. 28 (1972) 1421; M. S. Chanowitz and J. Ellis, Phys. Lett. B40 (1972) 397; J. C. Collins, A. Duncan, and S. D. Joglekar, Phys. Rev. D16 (1977) 438.

[23] G. Grunberg, Phys. Letts. B325 (1994) 441.

[24] M. A. Shifman, A. I. Vainshtein, and V. I. Zhakharov, Nucl. Phys. B147 (1979) 385, 448.

[25] V. A. Novikov et. al. Nucl. Phys. B249 (1985) 445.

[26] T. Kinoshita, Prog. Theor. Phys. 5 (1950) 1045; T. D. Lee and M. Nauenberg, Phys. Rev. 133B (1964) 1549.

[27] V. I. Zakharov, Nucl. Phys. B385 (1992) 452.

[28] R. K. Ellis, W. Furmanski, and R. Petronzio, Nucl. Phys. B212 (1983) 29.

[29] X. Ji, Nucl. Phys. B402 (1993) 217.

[30] X. Ji et al., to be published.

[31] H. J. Rothe, Lattice Gauge Theories: An Introduction, World Scientific, Singapore, 1992.

[32] The MIT lattice collaboration, to be published. 
[33] R. L. Jaffe and G. G. Ross, Phys. Letts. 93B (1980) 313.

[34] I. Hinchliffe, in Review of Particle Properties by Particle Data Group, Phys. Rev. D50 (1994) 1173. 


\section{FIGURES}

FIG. 1. a). A bubble-chain diagram for the coefficient function of the Bjorken sum rule. b). The corresponding matrix element of the twist-four operator.

FIG. 2. The complete one-loop Feynman diagram for the the coefficient function of the Bjorken sum rule. 
This figure "fig1-1.png" is available in "png" format from: http://arxiv.org/ps/hep-ph/9411312v1 